\title{
Nano-Scale Strain-Induced Giant Pseudo-Magnetic Fields and Charging Effects in CVD-Grown Graphene on Copper
}

Nai-Chang Yeh, Marcus L. Teague, Renee T. Wu, Sinchul Yeom, Brian Standley, David Boyd and Marc W. Bockrath

ECS Trans. 2011, Volume 35, Issue 3, Pages 161-172.

doi: $10.1149 / 1.3569908$

Email alerting

Receive free email alerts when new articles cite this article - sign up in the service box at the top right corner of the article or click here 


\title{
Nano-Scale Strain-Induced Giant Pseudo-Magnetic Fields and Charging Effects in CVD-Grown Graphene on Copper
}

\author{
N.-C. Yeh ${ }^{\mathrm{a}}$, M. L. Teague ${ }^{\mathrm{a}}$, R. T.-P. Wu ${ }^{\mathrm{a}}$, S. Yeom ${ }^{\mathrm{b}}$, B. L. Standley ${ }^{\mathrm{b}}$, D. A. Boyd ${ }^{\mathrm{b}}$, \\ and M. W. Bockrath ${ }^{\text {b,c }}$ \\ ${ }^{a}$ Department of Physics, California Institute of Technology, Pasadena, California 91125, \\ USA \\ ${ }^{b}$ Department of Applied Physics, California Institute of Technology, Pasadena, \\ California 91125, USA \\ ${ }^{\mathrm{c}}$ Department of Physics, University of California, Riverside, California 92521, USA
}

Scanning tunneling microscopic and spectroscopic (STM/STS) studies of graphene grown by chemical vapor deposition (CVD) on copper reveal that the monolayer carbon structures remaining on copper are strongly strained and rippled, with different regions exhibiting different lattice structures and local electronic density of states (LDOS). The large and non-uniform strain induces pseudomagnetic field up to $\sim 50$ Tesla, as manifested by the integer and fractional pseudo-magnetic field quantum Hall effects (IQHE and FQHE) in the LDOS of graphene. Additionally, ridges appear along the boundaries of different lattice structures, which exhibit excess charging effects. For graphene transferred from copper to $\mathrm{SiO}_{2}$ substrates after the CVD growth, the average strain and the corresponding charging effects and pseudo-magnetic fields become much reduced. Based on these findings, we consider realistic designs of strain-engineered graphene nano-transistors, which appear promising for nano-electronic applications.

The electronic properties of graphene exhibit significant dependence on the surrounding environment and high susceptibility to disorder because of the single layer of carbon atoms that behave like a soft membrane and because of the fundamental nature of Dirac fermions (1). In general, the sources of disorder in graphene may be divided into intrinsic and extrinsic disorder (1). Examples of intrinsic disorder include surface ripples and topological defects (1), whereas extrinsic disorder can come in many different forms, such as adatoms, vacancies, extended defects including edges and cracks, and charge in the substrate or on top of graphene (1).

There are two primary effects associated with disorder on the electronic properties of graphene (1). The first effect is a local change in the single site energy that leads to an effective shift in the chemical potential for Dirac fermions (1). One example of this type of disorder stems from charge impurities. The second type of disorder effect arises from changes in the distance or angles between the $p_{z}$ orbitals (1). In this case, the hopping energies (and thus hopping amplitudes) between different lattice sites are modified, leading to the addition of a new term to the original Hamiltonian. The new term results in the appearance of vector (gauge) $\mathscr{A}$ and scalar potentials $\phi$ in the Dirac Hamiltonian (1). The presence of a vector potential in the problem indicates that an effective magnetic field $B_{S}=\left(c / e v_{F}\right) \nabla \times \mathscr{C A}$ is also present, with opposite directions for the two inequivalent Dirac cones at $\mathrm{K}$ and $\mathrm{K}^{\prime}$ so that the global time-reversal symmetry is preserved. Here $v_{F}$ 
denotes the Fermi velocity of the Dirac fermions. Similarly, the presence of a spatially varying scalar potential can lead to local charging effects known as self-doping (1). One mechanism for inducing pseudo-magnetic fields and charging effects is strain (2-10), which is further elaborated below.

The strong susceptibility of graphene to external influences can in fact provide opportunities for engineering unique properties of graphene. For instance, it has been theoretically proposed that a designed strain aligned along three main crystallographic directions induces strong gauge fields that effectively act as a uniform pseudo-magnetic field on the Dirac electrons (1-3). This prediction was first verified empirically via scanning tunneling microscopy (STM) studies of graphene nano-bubbles grown on $\operatorname{Pt}(111)$ substrates, with relatively uniform pseudo-magnetic fields up to 600 Tesla over each graphene nano-bubble (8). More specifically, the strain-induced gauge potential $\mathscr{A}$ $=\left(\mathrm{A}_{\mathrm{x}}, \mathrm{A}_{\mathrm{y}}\right)$ may be related to the two-dimensional strain field $\boldsymbol{u}_{i j}(x, y)$ by the following relation (with $x$-axis along the zigzag direction) $(1,2)$ :

$$
\mathbf{A}=\frac{-(\partial \ln t / \partial \ln a)}{a}\left(\begin{array}{c}
u_{x x}-u_{y y} \\
-2 u_{x y}
\end{array}\right) \equiv \frac{\beta}{a}\left(\begin{array}{c}
u_{x x}-u_{y y} \\
-2 u_{x y}
\end{array}\right),
$$

where $t$ denotes the nearest hopping constant, $a$ represents the nearest carbon-carbon distance, $\beta$ is a constant ranging from 2 to 3 (1), and for a two-dimensional displacement field $\boldsymbol{u}=\left(u_{x}, u_{y}\right)$ we denote $u_{x x} \equiv\left(\partial u_{x} / \partial x\right), u_{x y} \equiv\left(\partial u_{x} / \partial y\right)$ and $u_{y y} \equiv\left(\partial u_{y} / \partial y\right)$. It is clear from Eq. [1] that any uniaxial strain would lead to a uniform gauge potential and therefore no pseudo-magnetic field.

In this work, we report our discovery of strained-induced giant pseudo-magnetic fields and charging effects in graphene grown by means of chemical vapor deposition (CVD) on copper (11). We attribute our findings to the result of large and non-uniform strain induced by the expansion of graphene and the contraction of the copper substrate upon cooling from the CVD-growth temperature $\left(\sim 1000^{\circ} \mathrm{C}\right)$ to low temperatures $(9,10)$. The strain-induced pseudo-magnetic fields give rise to discrete peaks in the electronic density of states at quantized energies that are consistent with the occurrence of quantum Hall effects in nano-scales (1-3), and the pseudo-magnetic fields in strongly strained regions are found to exceed 50 Tesla (9). Additionally, the strain-induced charging effects produce localized high conductance regions wherever maximum strains occur (9). Both the pseudo-magnetic fields and charging effects diminish after the CVD-grown graphene is transferred from the original copper substrate to a $\mathrm{SiO}_{2}$ substrate (9). Based on these findings, we propose realistic designs of strain-engineered graphene nano-transistors and examine the expected performance, and we find the results promising for nano-electronic applications.

\section{Experimental}

Our primary experimental tool for investigating the local electronic and structural correlations of graphene is a homemade cryogenic STM, which is compatible with high magnetic fields and also capable of variable temperature control from room temperature to $6 \mathrm{~K}$, with a vacuum level of $\sim 10^{-10}$ Torr at the lowest temperatures. For studies reported in this work, the measurements were made at $77 \mathrm{~K}$ and $300 \mathrm{~K}$ under high vacuum $\left(<10^{-7}\right.$ Torr) and in zero magnetic fields. Both topographic and spectroscopic 
measurements were performed simultaneously at every location in a $(128 \times 128)$ pixel grid. At each pixel location, the tunnel junction was independently established so that the junction resistance of $1.5 \mathrm{G} \Omega$ was maintained across the sample. The differential conductance, $(d I / d V)$, was calculated from the best polynomial fit of each current $(I)$ vs. bias voltage $(V)$ curve.

Our procedures for CVD growth of graphene on copper foils were largely consistent with the literature (11). Specifically, graphene films were primarily grown on $25-\mu \mathrm{m}$ thick $\mathrm{Cu}$ foils in a furnace consisting of a fused silica tube heated in a split tube furnace. The fused silica tube loaded with $\mathrm{Cu}$ foils was first evacuated and then back filled with hydrogen, heated to $\sim 1000^{\circ} \mathrm{C}$, and maintained under partial hydrogen pressure. A flow of $\mathrm{CH}_{4} \mathrm{was}$ subsequently introduced for a desired period of time at a total pressure of 500 mTorr. Finally, the furnace was cooled to room temperature, and the $\mathrm{Cu}$ foils coated with graphene appeared shiner relative to the as-received $\mathrm{Cu}$ foils, consistent with previous reports (11). The graphene sheets thus prepared were found to be largely single- or double-layered based on Raman spectroscopic studies. For the STM measurements of the CVD-grown graphene on copper, the sample was first cleaned and then loaded onto the STM probe. The STM probe was subsequently evacuated to high vacuum condition (< $10^{-7}$ Torr), and the sample was studied at both $\sim 77 \mathrm{~K}$ and room temperature. Both topographic and spectroscopic measurements were carried out.

To transfer CVD-grown graphene samples to $\mathrm{SiO}_{2}$ substrates, $\mathrm{SiO}_{2}$ substrates were prepared by first thermally grown a $290 \mathrm{~nm}$ thick $\mathrm{SiO}_{2}$ layer on the silicon wafer (p-type $\mathrm{h} 100 \mathrm{i}$ ), followed by gentle sonication of the substrate in acetone and then pure alcohol for about two minutes. The substrate was then baked at $115^{\circ} \mathrm{C}$ on a hotplate, nitrogen blown dry while cooling down. Next, a layer of PMMA was deposited on top of the CVDgrown graphene on copper as scaffolding, and then the copper substrate was removed with nitric acid. The PMMA/graphene sample was subsequently placed on a $\mathrm{SiO}_{2}$ substrate with the graphene side down. Finally, the PMMA was removed with acetone. The transferred graphene was cleaned and annealed in an argon atmosphere at $400^{\circ} \mathrm{C}$ for 30 minutes. Finally, electrodes were created by thermally evaporating $2.5 \mathrm{~nm}$ chromium and $37.5 \mathrm{~nm}$ gold through an aluminum foil shadow mask. Prior to STM measurements, the sample was rinsed again gently with acetone followed by pure alcohol to remove possible organic surface contaminants. The STM/STS studies on the transferred graphene sample on $\mathrm{SiO}_{2}$ were all conducted on the single-layer region.

\section{Results and Discussion}

We observed significant differences in the strain-induced effects between the CVDgrown graphene before and after transferred from $\mathrm{Cu}$ to $\mathrm{SiO}_{2}$, as detailed below.

\section{$\underline{\text { CVD-Grown Graphene on } \mathrm{Cu}}$}

The large ripples associated with the CVD-grown graphene on copper $(9,10)$ resulted in significant lattice distortion to the arrangements of carbon atoms. As exemplified in the atomically resolved topographic image in Fig. 1a, different atomic arrangements are apparent in different regions of the $(3.0 \times 3.0) \mathrm{nm}^{2}$ area in view. Specifically, we find that in a more relaxed region of the graphene sample (denoted as the $\alpha$-region in the upper 
portion of the topographic map) the atomic structure appeared to resemble the honeycomb or hexagon-like lattice with slight distortion. On the other hand, for spectra taken on the areas ( $\beta$-region) slightly below an apparent ridge, the atomic arrangements deviate strongly from those of graphene, showing either nearly square-lattice or disordered atomic structure in the $\beta$-region. Additionally, we find certain areas that are completely disordered without atomic resolution, as exemplified in Fig. 2a and denoted as the $\gamma$-region.

(a)

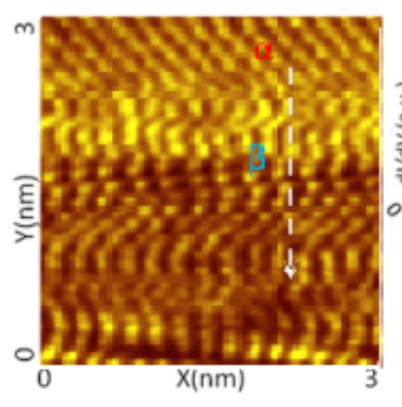

(b)

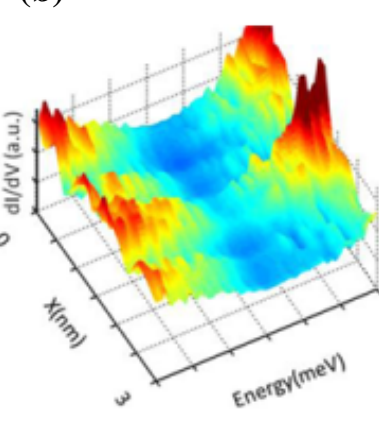

(c)

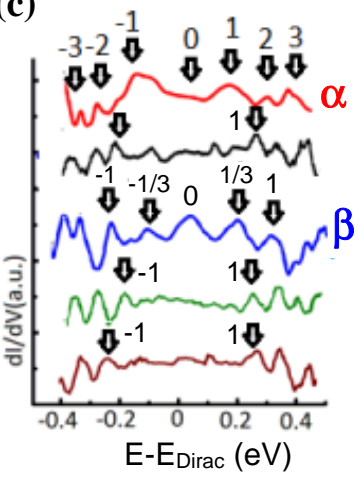

(d)

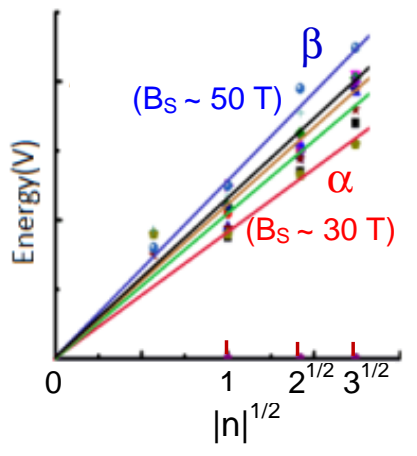

Figure 1. Strain-induced effects on the nano-scale structural and electronic properties of CVD-grown graphene on $\mathrm{Cu}$, taken with a cryogenic STM at $77 \mathrm{~K}$ : (a) A representative atomically resolved topography over a $(3.0 \times 3.0) \mathrm{nm}^{2}$ area, showing different atomic arrangements of the graphene sample in view, with slightly skewed honeycomb (or hexagonal) lattices in the $\alpha$-region and a combination of nearly squared and disordered atomic arrangements in the $\beta$-region. (b) Tunneling conductance $d I / d V$-vs.- $V$ spectra along the line-cut shown by the white dashed line in (a). (c) Representative tunneling conductance spectra after the subtraction of a smooth conductance background, showing quantized conductance peaks that are associated with strain-induced pseudo-magnetic fields $\left(B_{S}\right)$. (d) $\left(E-E_{\text {Dirac }}\right)-v s .-|n|^{1 / 2}$ for a number of spectra taken in the $\alpha$ and $\beta$ regions, showing that the data for each point spectrum follow a straight line whose slope it proportional to the pseudo-magnetic field $B_{S}$. The slopes of all $\left(E-E_{\text {Dirac }}\right)-v s .-|n|^{1 / 2}$ curves taken from different point spectra range from $B_{S} \sim 30$ Tesla for most point spectra in the $\alpha$-region to $B_{S} \sim 50$ Tesla for the strongest strained areas in the $\beta$-region.

To investigate the effect of lattice distortion on the local density of states (LDOS) of graphene, we compare the normalized tunneling conductance $(d I / d V) /(I / V)$-vs.- $V$ spectra obtained from different regions of atomic arrangements. As shown in Fig. 1b for a series of tunneling spectra along the dashed line indicated in Fig. 1a, we observe apparent spatial variations in the spectra that depend sensitively on the local strain. Moreover, we notice sharp conductance peaks in the tunneling spectra. If we subtract off a parabolic conductance background due to the copper contribution (indicated by the dashed lines in Fig. $1 b)$ and plot the resulting conductance against $\left(E-E_{\text {Dirac }}\right)$ where $E_{\text {Dirac }}$ is the Dirac energy, we find distinct peaks occurring at energies proportional to $|n|^{1 / 2}$ for $n=0, \pm 1, \pm 2$, \pm 3 , as exemplified in Fig. 1c. Furthermore, for $\beta$-type spectra, sharp peaks at energies proportional to $\operatorname{sgn}(n)|n|^{1 / 2}$, such as those proportional to $\pm(1 / 3)^{1 / 2},-(2 / 3)^{1 / 2}$ and $(5 / 3)^{1 / 2}$, are clearly visible. These peaks are in contrast to the weak "humps" occurring in the $\alpha$ type spectrum at energies proportional to $(1 / 3)^{1 / 2}$ and $-(5 / 3)^{1 / 2}$. By plotting $\left(E-E_{\text {Dirac }}\right)$ vs. $|n|^{1 / 2}$ data for multiple spectra taken in both the $\alpha$ and $\beta$-regions in Fig. 1d, we find that 
for every point spectrum, the energies at which the conductance peaks occur all fall approximately on one linear curve in the $\left(E-E_{\text {Dirac }}\right)$ vs. $|n|^{1 / 2}$ plot, although the slope varies from point to point. In general, the slopes for the $\beta$-type spectra are larger than those for the $\alpha$-type spectra.

The results manifested in Figs. 1c $-1 \mathrm{~d}$ can be understood in terms of the presence of strain-induced pseudo-magnetic fields $B_{S}$, with larger strain giving rise to larger $B_{S}$ values $(2,3,8,9)$. Specifically, the pseudo-Landau levels $E_{n}$ of Dirac electrons under a given $B_{S}$ satisfy the following relation:

$$
E_{n}=\operatorname{sgn}(n) \sqrt{2 e v_{F}^{2} \hbar B_{S}|n|},
$$

where $n$ denotes either integers or fractional numbers. Therefore, the slope of $\left(E-E_{\text {Dirac }}\right)$ vs. $|n|^{1 / 2}$ for each point spectrum is directly proportional to $\left(B_{S}\right)^{1 / 2}$. Using $v_{F} \sim 10^{6} \mathrm{~m} / \mathrm{s}$, we find that the pseudo-magnetic fields range from approximately 29 Tesla for most spectra in the $\alpha$-region to 50 Tesla for most strained areas in the $\beta$-region.

Although these $B_{S}$ values are smaller than those reported for graphene nano-bubbles (8) and are also inhomogeneous, we note that the conductance peaks associated with the CVD-grown graphene are in fact more distinct, being clearly visible even at a relatively high temperature of $77 \mathrm{~K}$ as opposed to the weaker speaks associated with individual graphene nano-bubbles at $4.2 \mathrm{~K}$. Moreover, the $B_{S}$ values for the CVD-grown graphene on $\mathrm{Cu}$ are inhomogeneous in space due to complicated strain distributions, which is in sharp contrast to the nearly homogeneous $B_{S}$ values in graphene nano-bubbles. In particular, pronounced fractional $n$-values in addition to the integer $n$-values are observed for the first time in the strongly strained $\beta$-region, implying pseudo-magnetic fieldinduced fractional quantum Hall effect (FQHE) besides the integer quantum Hall effect (IQHE). The occurrence of strain-induced FQHE in CVD-grown graphene may be due to the need of including more complicated gauge potentials of the Chern-Simons type in the Hamiltonian besides the simple gauge potential given in Eq. [1] in order to fully account for the behavior of Dirac fermions under very strong and inhomogeneous strain. This situation is similar to the FQHE in two-dimensional electron systems (2DES) induced by strong external magnetic fields. In the latter case the FQHE must be described in terms of the effective Chern-Simons theory (12-15), which is in addition to the simple vector potential associated with the external magnetic field that can only account for the IQHE. However, we note that to date there have not been self-consistent theoretical calculations for strain-induced FQHE.

The aforementioned strain-induced pseudo-magnetic fields and IQHE reflect the unique characteristics of the Dirac fermions in graphene, namely, the massless linear energy dispersion relation. This is in stark contrast to the massive and parabolic energy dispersion relation found in semiconducting 2DES. On the other hand, for the amorphous regions of the CVD-grown graphene sample shown in Fig. 2, we find that the spectral characteristics differ fundamentally from those of Dirac fermions. As exemplified in Figs. $2 \mathrm{~b}$ and $2 \mathrm{c}$, most point spectra in the amorphous $\gamma$-region are largely smooth and parabolic in energy, which suggests that the characteristics of conduction carriers in amorphous graphene differ fundamental from the behavior of Dirac fermions. However, further theoretical studies would be necessary to shed light on this finding. 
(a)

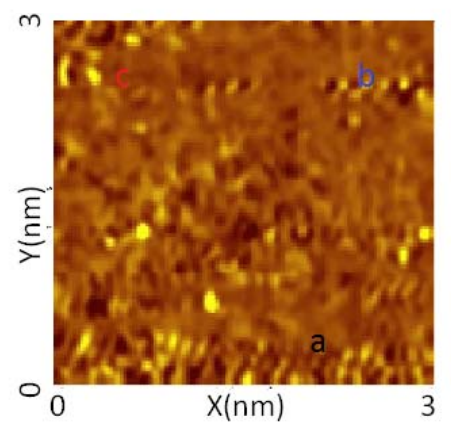

(b)

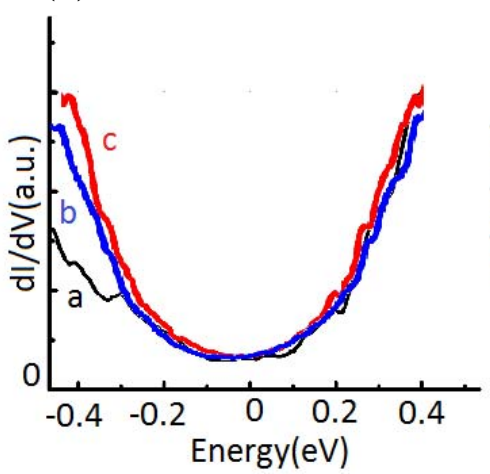

(c)

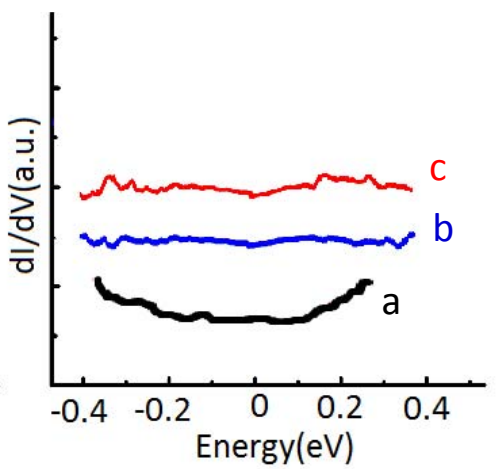

Figure 2. Strain-induced strong lattice distortion in CVD-grown graphene on $\mathrm{Cu}$, leading to amorphous structures in certain areas: (a) Topography of a representative $(3.0 \times 3.0) \mathrm{nm}^{2}$ area, showing amorphous behavior in the $\gamma$-region. (b) $(d I / d V) /(I / V)-v s .-V$ point spectra at three locations denoted by a, b, c in (a). All spectra exhibit a parabolic background and are relatively smooth. (c) The three tunneling spectra in (b) after the subtraction of a smooth conductance background. Here the spectra are shifted vertically for clarity. All data were taken at $77 \mathrm{~K}$.

\section{$\underline{\text { CVD-Grown Graphene Transferred from } \mathrm{Cu} \text { substrate to } \mathrm{SiO}_{2}}$}

We may further verify the effect of strain on the electronic properties of graphene by conducting a comparative study of CVD-grown graphene transferred from $\mathrm{Cu}$ to $\mathrm{SiO}_{2}$. In general we find that the height variations and the corresponding strain in the transferred graphene samples are much reduced (9), as exemplified in Fig. 3a. Hence, the conductance spectra for most areas of the sample are quite smooth without quantized conductance peaks, as exemplified in Fig. 3b. However, occasional quantized LDOS spectra are still present in regions that appear to be remnant "ridges" of the CVD-grown graphene, as shown in Fig. 3c. These quantized peaks still obey the general relation ( $E^{-}$ $\left.E_{\text {Dirac }}\right) \propto|n|^{1 / 2}$ except that the slopes are much smaller, implying much suppressed pseudomagnetic fields, (Fig. 3d). This is consistent with the significant reduction in strain upon transferring the graphene sample from $\mathrm{Cu}$ to $\mathrm{SiO}_{2}$.

\section{Estimates of the Strain Fields}

In contrast to the relatively weak lattice distortion in exfoliated graphene on $\mathrm{SiO}_{2}$ substrates $(4,10)$, the severe nano-scale lattice distortion exemplified in Fig. 1a makes it difficult to compute the resulting strain fields directly. Nonetheless, we may estimate the magnitude of strain in a severely distorted graphene sample by considering the strain induced by corrugations along the out of plane direction. In this case, the typical strain $\langle|\boldsymbol{u}|\rangle$ is of order of the square of the height variation $l$ over a strained region $L,\langle|\boldsymbol{u}|\rangle \sim$ $(I / L)^{2}(2,16,17)$. Hence, the relative spatial variations of the strain may be obtained from the topography $z(x, y)$ if we plot maps of $[\partial z(x, y) / \partial x]^{2},[\partial z(x, y) / \partial y]^{2}$ and $\left[(\partial z / \partial x)^{2}+(\partial z / \partial y)^{2}\right]$.

As illustrated in Figs. 4a-4c for the maps of $[\partial z(x, y) / \partial y]^{2},[\partial z(x, y) / \partial x]^{2}$ and the quantity $\left[(\partial z / \partial x)^{2}+(\partial z / \partial y)^{2}\right]$ over the same area shown in Fig. 1a, the maximum strain occurs right below the ridge that separates the $\alpha$ - and $\beta$-regions, consistent with our finding of maximum pseudo-magnetic fields and most pronounced $\mathrm{FQHE}$ in this area. Additional 
large strain regions also appear in the lower section of the map, which correspond to areas between two other lower ridge-like features.

(a)

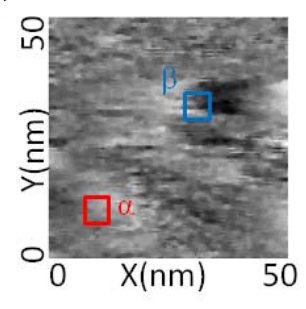

(b)

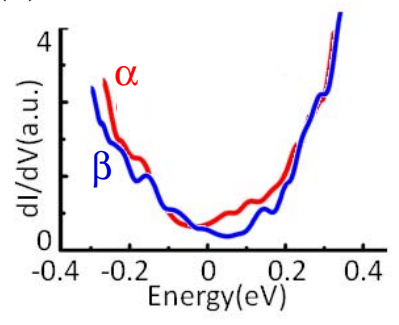

(c)

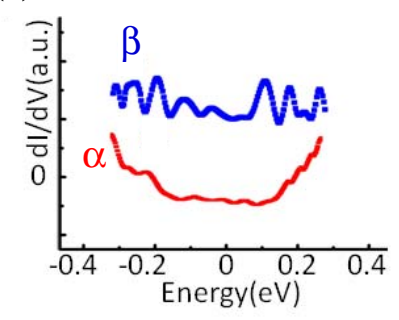

(d)

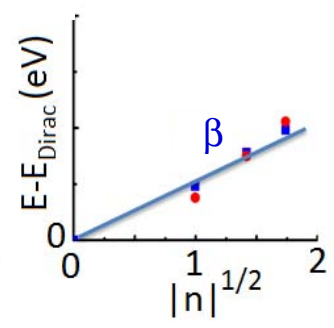

Figure 3. Topography and spectroscopy of a CVD-grown graphene sample transferred from $\mathrm{Cu}$ to $\mathrm{SiO}_{2}$ : (a) A representative topography over a $(50 \times 50) \mathrm{nm}^{2}$ area, showing mostly flat regions (represented by the $\alpha$-region) as well as regions containing ridges (represented by the $\beta$-region) that are associated with the rippling phenomena of the CVD-grown graphene. (b) Representative tunneling conductance spectra of the $\alpha$ - and $\beta$ regions, showing smooth graphene-like spectra in the $\alpha$-regions and sharp peaks at the $\beta$ region. (c) Representative tunneling conductance spectra after the subtraction of a smooth conductance background, showing strain-induced quantized conductance peaks at quantized energies with $|n|=1,2,3$ only in the $\beta^{\prime}$-region. (d) $\left(E-E_{\text {Dirac }}\right)$-vs.- $|n|^{1 / 2}$ for spectra taken in the $\beta$-region, showing that the conductance peaks fall on one linear curve that corresponds to $B_{S}=8 \pm 1$ Tesla.

(a) $[\partial z(x, y) / \partial y]^{2}$

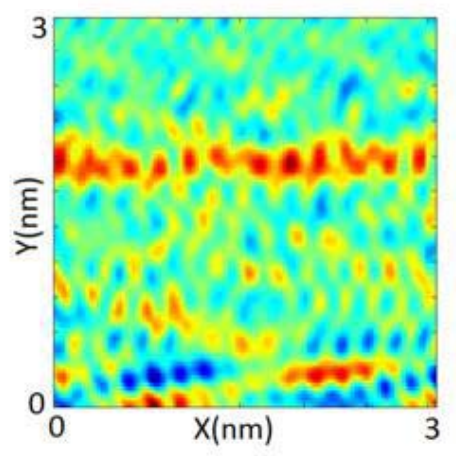

(b) $\quad[\partial z(x, y) / \partial x]^{2}$

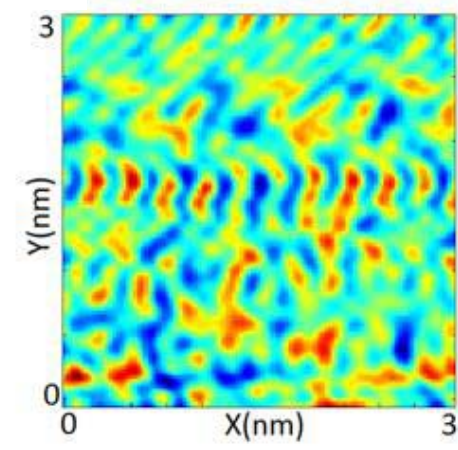

(c) $\left[(\partial z / \partial x)^{2}+(\partial z / \partial x)^{2}\right]$

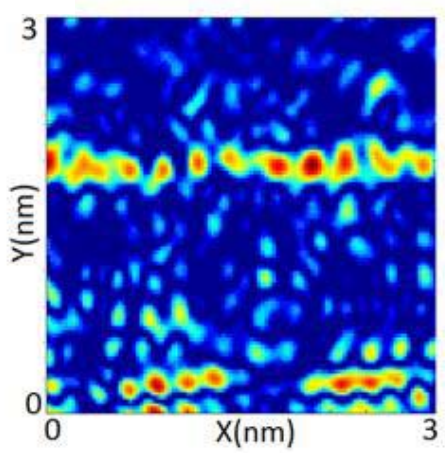

Figure 4. Estimate of the spatial variations of the typical strain $\langle|\boldsymbol{u}|\rangle$ over the same $(3.0 \times 3.0) \mathrm{nm}^{2}$ area shown in Fig. 1a: (a) $[\partial z(x, y) / \partial y]^{2}$ map, (b) $[\partial z(x, y) / \partial x]^{2}$ map, and (c) $\left[(\partial z / \partial x)^{2}+(\partial z / \partial y)^{2}\right] \sim\langle|\boldsymbol{u}|\rangle$ map, showing maximum strain immediately below the ridge in the upper half of the scanned region and additional strained areas in the lower section of the map that situates between two lower ridge-like features.

\section{$\underline{\text { Strain-Induced Charging Effects }}$}

The strain found in the CVD-grown graphene on copper is not purely shear but also contains dilation/compression components. The latter is theoretically predicted to gives rise to an effective scalar potential $V(x, y)$ and therefore a static charging effect $(3,16,17)$ 
in addition to the aforementioned pseudo-magnetic field. The scalar potential $V(x, y)$ is given by $(3,16,17)$ :

$$
V(x, y)=V_{0}\left(u_{x x}+u_{y y}\right)=V_{0}\langle|\boldsymbol{u}|\rangle
$$

where $V_{0} \sim 3 \mathrm{eV}$, and $\langle|\boldsymbol{u}|\rangle$ is the dilation/compression strain. While the charging effect may be largely screened if the height variation $l$ is much smaller than the magnetic length $l_{B} \equiv\left[\Phi_{0} /\left(2 \pi \mathrm{B}_{S}\right)\right]^{1 / 2}$, where $\Phi_{0}$ is the flux quantum (1-3), we note that for $B_{S}$ ranging from 30 to 50 Tesla, the corresponding $l_{B}$ ranges from $5.5 \mathrm{~nm}$ to $3.5 \mathrm{~nm}$. Given that the height variation $l$ over the sample area shown in Fig. 1a is on the order of $1 \mathrm{~nm}$, comparable to the magnetic length, we expect significant charging effect in the CVD-grown graphene on $\mathrm{Cu}$.

To visualize the charging effect, we show in Figs. $5 \mathrm{a}-5 \mathrm{f}$ the conductance maps at different constant bias voltages over the same $(3.0 \times 3.0) \mathrm{nm}^{2}$ area. The bias voltages are chosen to represent the pseudo-Landau levels of the $\beta$-type spectra for $n=0,1 / 3,2 / 3,1,2$, 3 . A nearly one-dimensional high-conductance region close to the most strained region immediately below the ridge is clearly visible for maps associated with smaller $n$ values, confirming the notion of strain-induced charging effects. For higher energies (larger $n$ values), the high-conductance region becomes less confined, which is reasonable because of the higher confinement energies $\left(\propto|n|^{1 / 2}\right)$ required for the Dirac electrons.
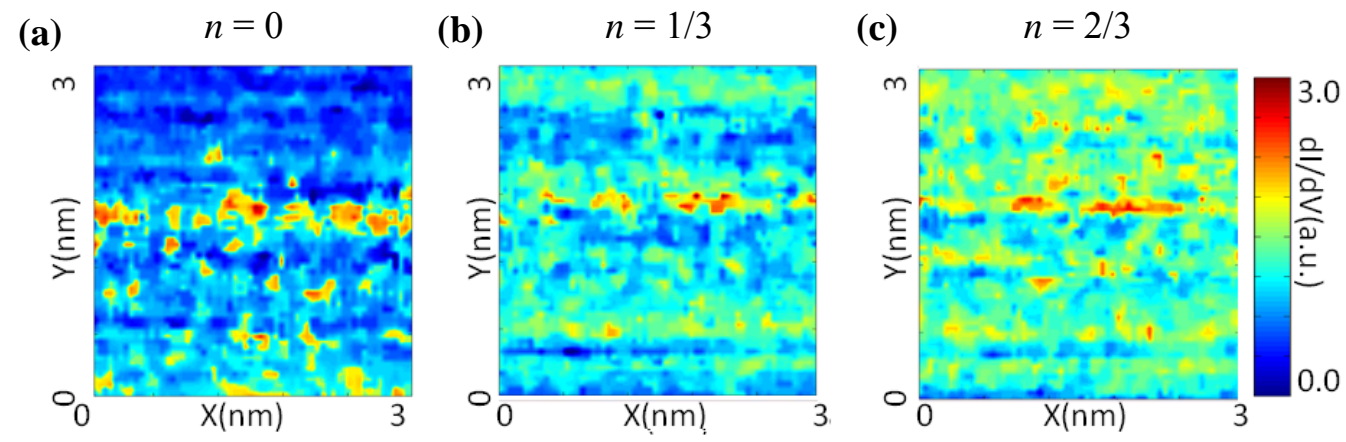

(d)

(e)
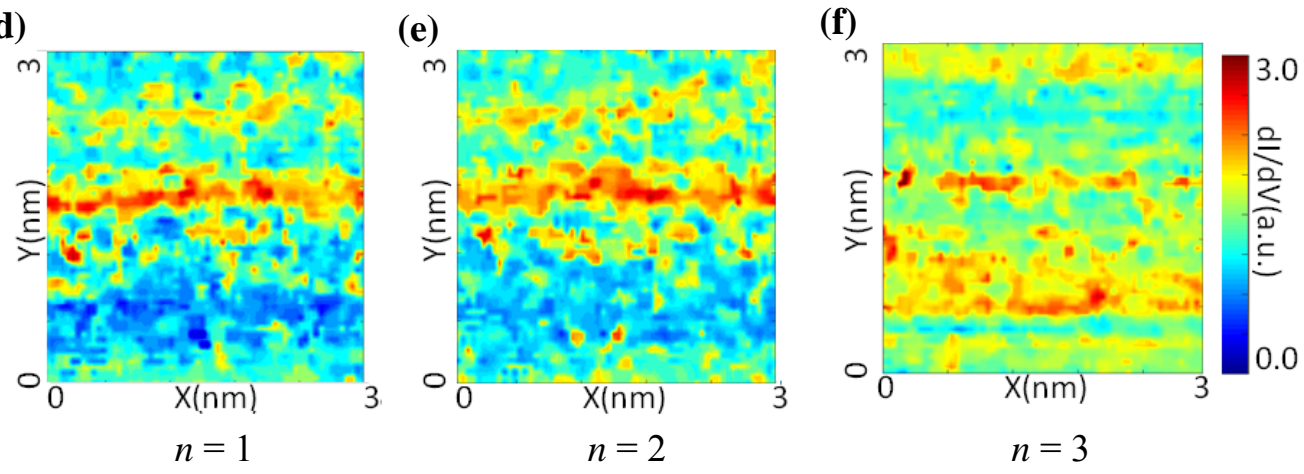

Figure 5. Constant-voltage tunneling conductance maps at quantized energies of the pseudo-Landau levels over a $(3.0 \times 3.0) \mathrm{nm}^{2}$ area and for $T=77 \mathrm{~K}$ : (a) $n=0$, (b) $n=1 / 3$, (c) $n=2 / 3$, (d) $n=1$, (e) $n=2$, (f) $n=3$, where the $n$ values are referenced to the $\beta$-type spectra with $B_{S} \sim 50$ Tesla. For smaller $n$ values, an approximately one-dimensional highconductance "line" appears near the topographical ridge where the most abrupt changes in height occur, suggesting significant charging effects. The confinement of highconductance region diminishes with increasing $n$. 
The Fourier transformation (FT) of the constant-energy conductance maps for integer $n$ values are given in Figs. $6 \mathrm{a}-6 \mathrm{~d}$. We find that for $n$ equals to an even integer, the FTLDOS map exhibits sharper diffraction spots, implying better defined characteristic wave-vectors for the Dirac fermions, which in contrast to the broader distributions of the spectral weights and therefore more diffusive motion of Dirac fermions for $n$ equals to an odd integer. The physical origin for the spectral differences in the FT-LDOS maps for varying $n$ values remains unknown and awaits further theoretical investigations.

(a) $\quad n=0$

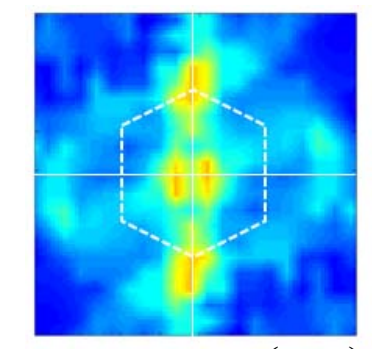

(b) $n=1$

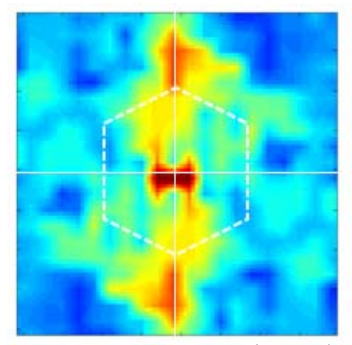

(c) $n=2$

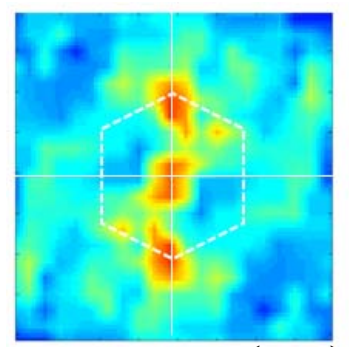

(d) $n=3$

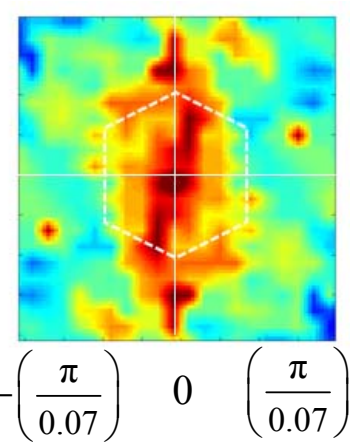

Figure 6. Fourier-transformation (FT) of the conductance maps in Fig. 5 is shown in the reciprocal space, with the white hexagon representing the first Brillouin zone of graphene: (a) $n=0$, (b) $n=1$, (c) $n=2$, (d) $n=3$, where the $n$ values are referenced to the $\beta$-type spectra with $B_{S} \sim 50$ Tesla, and the units for the $k_{x}$ and $k_{y}$ axes are in $(\mathrm{nm})^{-1}$. For $n$ equals to an even integer, the FT-LDOS map exhibits sharper spots, implying better defined wave-vectors for the Dirac fermions. In contrast, more extended momentum distributions appear for $n$ equals to an odd integer, suggesting more diffusive motion of the Dirac fermions.

\section{Discussion}

Having established the existence of strain-induced pseudo-magnetic fields at the nanoscale, we compare the resulting electrical transport characteristics with those induced by real magnetic fields. This quantitative comparison is essential to assessing the feasibility of strain-engineered graphene nano-devices for electronic applications. For realistic strained graphene devices, we may develop arrays of nano-dots with optimized geometry on the substrates for graphene so that the strain thus induced corresponds to large and uniform pseudo-magnetic fields on each nano-dot, similar to the situation demonstrated for the graphene nano-bubbles (8).

In Figs. $7 \mathrm{a}-7 \mathrm{~d}$ we consider the electrical transport properties (longitudinal conductivity $\sigma_{x x}$, Hall conductivity $\sigma_{x y}$, longitudinal resistivity $\rho_{x x}$, and Hall resistivity $\rho_{x y}$ ) of graphene under a uniform external magnetic field $B=14$ Tesla and as a function of the gated voltage, following the empirical results in Ref. (18). For comparison, we consider a uniform strain-induced pseudo-magnetic field of $B_{S}=50$ Tesla and simulate the corresponding $\sigma_{x x}, \sigma_{x y}, \rho_{x x}$, and $\rho_{x y}$ vs. energy $(E)$ in Figs. 7e-7h. Based on the simulated results, we note that a sizable on/off resistance ratio can be achieved by engineering the strained graphene into a transistor. Specifically, using the simulated results for $\rho_{x x}$-vs.-E 
in Fig. $7 \mathrm{~g}$, we obtain a sizable on/off source-drain resistance ratio $\left[\left(\rho_{x x}\right)_{\text {on }} /\left(\rho_{x x}\right)_{\text {off }}\right]>10$ for $E_{\text {on }}=0$ and $E_{1}<E_{\text {off }}<E_{2}$, where $E_{n}$ with $n=1,2$ follows the relation in Eq. [2]. The advantages of such strain-engineered graphene nano-transistors include the smallness of these nano-devices and the fact that the switching voltages and the magnitude of $\left[\left(\rho_{x x}\right)_{\text {on }} /\left(\rho_{x x}\right)_{\text {off }}\right]$ can be designed geometrically by controlling the surface texture of the substrate for graphene.

(a)

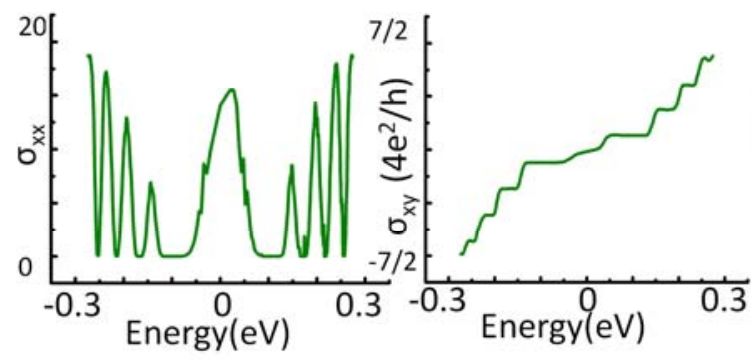

(e)

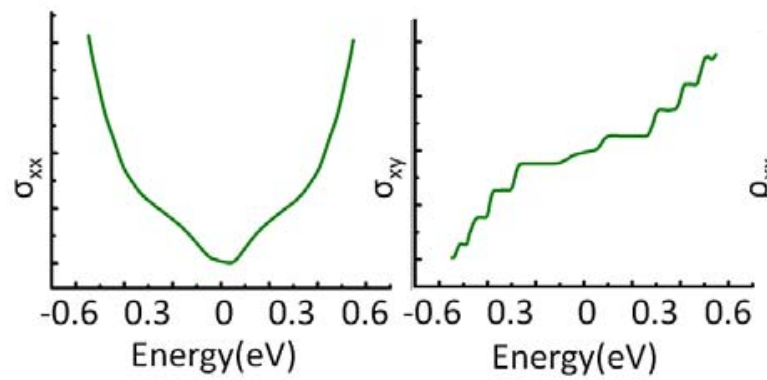

(c)

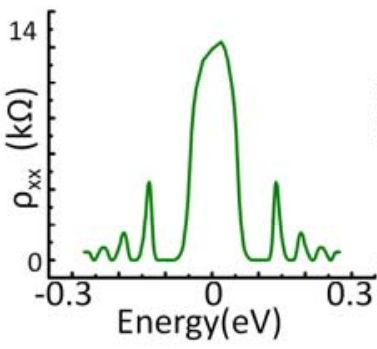

(g)

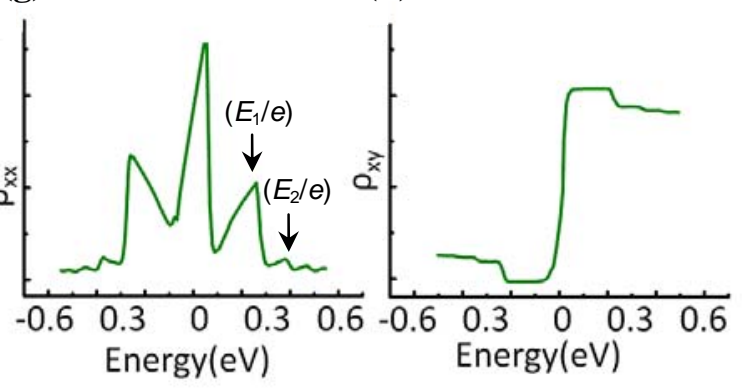

Figure 7. Comparison of the energy-dependent electrical transport properties of graphene under a real magnetic field $B\left((\mathrm{a})\right.$ - (d)) and a strain-induced pseudo-magnetic field $B_{S}$ ((e) - (h)): (a) longitudinal conductivity $\sigma_{x x}$, (b) Hall conductivity $\sigma_{x y}$, (c) longitudinal resistivity $\rho_{x x}$ and (d) Hall resistivity $\rho_{x y}$ for $B=14$ Tesla. (e) Longitudinal conductivity $\sigma_{x x}$, (f) Hall conductivity $\sigma_{x y}$, (g) longitudinal resistivity $\rho_{x x}$ and (h) Hall resistivity $\rho_{x y}$ for $B_{S}=50$ Tesla. We note that the sharp $\rho_{x x}$ peak at the Dirac point of the strained graphene may be employed to produce a significant on/off ratio in a transistor. More specifically, the source-drain resistance of a graphene nano-transistor may be controlled by the gate voltage, and a large resistance ratio $\left[\left(\rho_{x x}\right)_{\text {on }} /\left(\rho_{x x}\right)_{\text {off }}\right]>\sim 10$ can be achieved for gate voltages switching between $E_{\text {on }}$ and $E_{\text {off }}$, with $E_{\text {on }}=0$ and $E_{1}<E_{\text {off }}<E_{2}$, where $E_{n}$ with $n$ $=1,2$ follows the relation in Eq. [2]. For $B_{S}=50$ Tesla and $v_{F}=10^{5} \mathrm{~m} / \mathrm{s}$, we have $E_{1} \sim$ $0.25 \mathrm{eV}$.

For realistic implementation of strained graphene nano-transistors, we need to consider an assembly of arrays of such transistors, which may be considered as a network of serial and parallel connections of both strained and unstrained graphene resistors. In Fig. 8a we simulate the $\rho_{x x}$-vs.- $V_{g}$ data ( $V_{g}$ being the gate voltage) for a serial connection of equally weighted strained and unstrained graphene resistors, and in Fig. $8 \mathrm{~b}$ we consider the $\rho_{x x^{-}}$ vs.- $V_{g}$ data for the parallel connection of two sets of graphene resistors in Fig. 8a. We find that the overall $\left[\left(\rho_{x x}\right)_{\text {on }} /\left(\rho_{x x}\right)_{\text {off }}\right]$ ratio is better preserved in the serial connections, whereas parallel connections tend to reduce the on/off ratios. Overall, the optimal straininduced effects can be achieved by maximizing the weight associated with the strained 
graphene. Furthermore, the fabrication of arrays of nano-dots on the substrates for straining graphene can be achieved by means of either standard nano-lithography or selfassembly techniques (19-21).

(a)

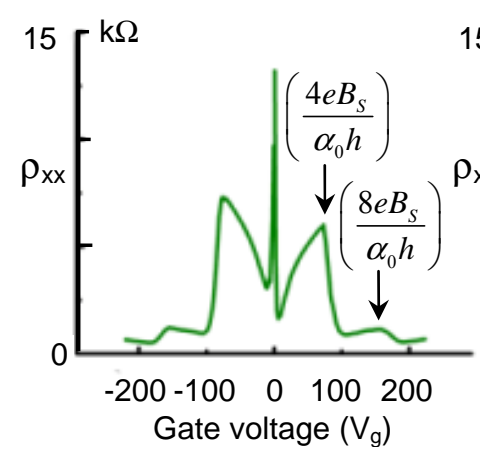

(b)

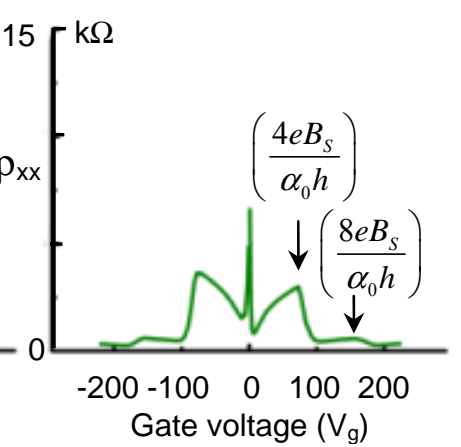

(c)

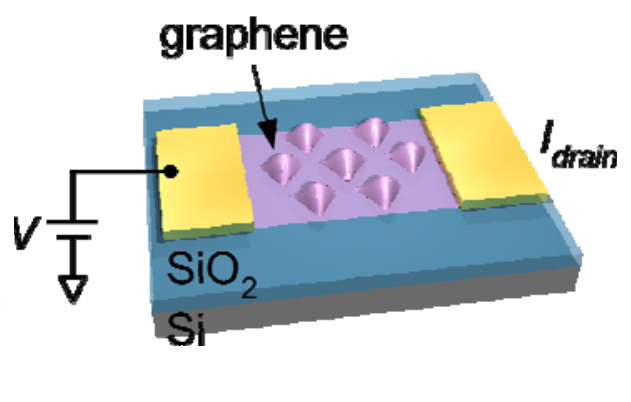

Figure 8. Modeling the device performance of arrays of strain-engineered graphene nano-transistors embedded in unstrained graphene: (a) $\rho_{x x}-\mathrm{vs} .-V_{g}$ characteristics for a serial connection of two equally weighted strained and unstrained graphene resistors with resistivity $\left(\rho_{x x}\right)_{S}$ and $\left(\rho_{x x}\right)_{0}$, respectively, so that $\rho_{x x}=0.5\left(\rho_{x x}\right)_{S}+0.5\left(\rho_{x x}\right)_{0}$, and the resistivity ratio $\left[\left(\rho_{x x}\right)_{\text {on }} /\left(\rho_{x x}\right)_{\text {off }}\right] \sim 7$ for $V_{\text {on }}=0$ and $4 e B_{S} /\left(\alpha_{0} h\right)<V_{\text {off }}<8 e B_{S} /\left(\alpha_{0} h\right)$ where $h$ is the Planck constant, and we have assume $B_{S}=50$ Tesla, and the coefficient $\alpha_{0}=7.3 \times$ $10^{14} \mathrm{~m}^{-2} \mathrm{~V}^{-1}$ is defined by the linear relationship between the gate voltage and the twodimensional carrier density $n_{2 \mathrm{D}}=\alpha_{0} V_{g}$. (b) Parallel connections of two sets of structures shown in (a) so that $\rho_{x x}=0.25\left(\rho_{x x}\right)_{S}+0.25\left(\rho_{x x}\right)_{0}$, and the resistivity ratio $\left[\left(\rho_{x x}\right)_{\text {on }} /\left(\rho_{x x}\right)_{\text {off }}\right] \sim$ 4 for $V_{\text {on }}=0$ and $4 e B_{S} /\left(\alpha_{0} h\right)<V_{\text {off }}<8 e B_{S} /\left(\alpha_{0} h\right)$ for $B_{S}=50$ Tesla. (c) Schematics of a graphene nano-transistor with graphene laid over periodic arrays of triangular nano-dots to optimize the strength and uniformity of the strain-induced pseudo-magnetic fields.

\section{Conclusion}

We have conducted spatially resolved topographic and spectroscopic studies of CVDgrown graphene on copper and CVD-grown graphene transferred to $\mathrm{SiO}_{2}$. Our investigation reveals the important influence of the substrate and strain on the carbon atomic arrangements and the electronic DOS of graphene. For CVD-grown graphene remaining on copper, the monolayer carbon structures exhibit strongly distorted, with different regions exhibiting varying lattice structures and electronic LDOS. In particular, topographical ridges appear along the boundaries between different lattice structures, giving rise to excess charging effects. Additionally, the large and non-uniform strain induces pseudo-magnetic fields up to $\sim 50$ Tesla, as manifested by quantized conductance peaks associated with the integer and fractional quantum Hall effects (IQHE/FQHE). In contrast, for graphene transferred from copper to $\mathrm{SiO}_{2}$ after CVD growth, the average strain on the whole is reduced, so are the corresponding charging effects and pseudomagnetic fields, except for limited regions containing remnant topological defects. Our findings suggest the feasibility of strain engineering of graphene by proper design of the surface textures of the substrates. Examples of realistic nano-transistors based on strained graphene nano-structures are examined and are found to be promising for nano-electronic applications. 


\section{Acknowledgments}

The work at Caltech was jointly supported by the National Science Foundation and the Nano Research Initiatives (NRI) under the Center of Science and Engineering of Materials (CSEM).

\section{References}

1. A. H. Castro Neto, F. Guinea, N. M. R. Peres, K. S. Novoselov, A. K. Geim, Rev. Mod. Phys., 81, 109 (2009).

2. F. Guinea, M. I. Katsnelson, A. K. Geim, Nat. Phys., 6, 30 (2010).

3. F. Guinea, M. I. Katsnelson, M. A. H. Vozmediano, Phys. Rev. B, 77, 075422 (2008).

4. M. L. Teague, A. P. Lai, J. Velasco, C. R. Hughes, A. D. Beyer, M. W. Bockrath, C. N. Lau, N.-C. Yeh, Nano Lett., 9, 2542 (2009).

5. G. M. Rutter, J. N. Crain, N. P. Guisinger, T. Li, P. N. First, J. A. Stroscio, Science, 317, 219 (2007).

6. C. L. Kane, E. J. Mele, Phys. Rev. Lett., 95, 226801 (2005).

7. B. A. Bernevig, S. C. Zhang, Phys. Rev. Lett., 96, 106802 (2006).

8. N. Levy, S. A. Burke, K. L. Meaker, M. Panlasigui, A. Zettl, F. Guinea, A. H. Castro Neto, M. F. Crommie, Science, 329, 544 (2010).

9. N.-C. Yeh, M. L. Teague, D. A. Boyd, M. W. Bockrath, J. Velasco, C.-N. Lau, ECS Transactions, 28 (5), 115 (2010).

10. N.-C. Yeh, M. L. Teague, S. Yeomb, B. L. Standleyb, R. T.-P. Wua, D. A. Boydb, M. W. Bockrath, to appear in Surface Science (2011); [arXiv:1009.0081].

11. X. Li, W. Cai, J. An, S. Kim, J. Nah, D. Yang, R. Piner, A. Velamakanni, I. Jung, E. Tutuc, S. K. Banerjee, L. Colombo, R. S. Ruoff1, Science, 324, 1312 (2009).

12. F. D. M. Haldane, Phys. Rev. Lett., 51, 605 (1983).

13. B. I. Halperin, Phys. Rev. Lett., 52, 1583 (1984).

14. B. Blok and X.-G. Wen, Phys. Rev. B, 42, 8133 (1990).

15. B. Blok and X.-G. Wen, Phys. Rev. B, 42, 8145 (1990).

16. H. Suzuura, T. Ando, Phys. Rev. B, 65, 235412 (2002).

17. J. L. Manes, Phys. Rev. B, 76, 045430 (2007).

18. K. S. Novoselov, A. K. Geim, S. V. Morozov, D. Jiang, M. I. Katsnelson, I. V. Grigorieva, S. V. Dubonos, and A. A. Firsov, Nature, 438, 197 (2005).

19. P. W. K. Rothemund, Nature, 440, 297 (2006).

20. S. M. Douglas, H. Dietz, T. Liedl, B. Hogberg, F. Graf, and W. M. Shih, Nature, 459, 414 (2009).

21. H. Dietz, S. M. Douglas, and W. M. Shih, Science, 325, 725 (2009). 